\title{
Tesis de Maestría
}

\section{Docencia de la Química}

Departamento de Química, Universidad Pedagógica Nacional

\section{Conceptos alternativos de profesores de química en formación, con respecto a cinética química y estrategia de enseñanza y aprendizaje}

\author{
Leonardo Fabio Martínez Pérez
}

\section{Descripción}

En esta tesis se presentan los resultados de investigación obtenidos con respecto a los conceptos alternativos que poseen los profesores de química en formación frente a conceptos de cinética química. A partir de esto se propone una estrategia didáctica, orientada por principios científicos-epistemológicos, psicológicos y didácticos, de acuerdo con el modelo de enseñanza y aprendizaje por investigación. Así mismo, en la estrategia se contempla el diseño de videos que contribuyen a un mejor aprendizaje de conceptos de cinética química.

\section{Contenidos}

En la primera parte de la tesis se muestra la importancia de contribuir a la Formación Inicial de Profesores de Química (FIPQ), mediante la implementación de estrategias de enseñanza y aprendizaje que favorezcan en los estudiantes una mejor apropiación conceptual de las temáticas fundamentales de la química, como es el caso de la cinética química; para esto, es clave que el profesor en formación maneje las estructuras conceptuales y metodológicas de la disciplina que va a enseñar, y que iden- tifique los obstáculos que ha implicado su construcción. A continuación, en el marco teórico se muestran los fundamentos científicos-epistemológicos, psicológicos y didácticos que sustentan la investigación.

A continuación se presenta la metodología de tipo cuantitativo y cualitativo que está conformada por dos etapas. Finalmente, se señala que los conceptos alternativos de los PFIQ dificultan el aprendizaje de los conceptos centrales de la cinética química. Así mismo, se muestra que una estrategia de enseñanza y aprendizaje, elaborada de acuerdo con el modelo de enseñanza y aprendizaje por investigación, contribuye significativamente al aprendizaje de los estudiantes.

\section{Metodología}

La metodología de la tesis consta de dos etapas. En la primera se caracterizan los conceptos alternativos de los PFIQ con respecto a los conceptos básicos de cinética química, mientras que en la segunda etapa, a partir de dichos conceptos y de los presupuestos teóricos, se diseña, implementa y evalúa la estrategia 
didáctica que posibilita la evolución del conocimiento por parte de los PFIQ.

\section{Conclusiones}

En la tesis se caracterizan algunos conceptos alternativos de los PFIQ con respecto a cinética química, entre los que se destacan los siguientes:

- Considerar que a medida que transcurre la reacción, la variación de concentración por unidad de tiempo de reactivos aumenta y la variación de concentración por unidad de tiempo de productos disminuye. Los PFIQ asocian el orden de reacción al coeficiente estequiométrico de los reactantes de la ecuación química.
- Considerar que la concentración de reactivos es inversamente proporcional a la velocidad de reacción. En el proceso de catálisis heterogénea se piensa que cuando el catalizador y el reactivo interactúan, el catalizador se descompone, desdobla, reduce o almacena el reactivo.

Con base en estos conceptos alternativos y los presupuestos teóricos se diseñó, implementó y evaluó una estrategia de enseñanza y aprendizaje que favorece un mejor aprendizaje en los PFIQ. Dicha estrategia implica un material educativo (módulo didáctico) y tres videos. $\mathbf{\Delta}$

\section{Del modelo del flogisto al modelo de la oxidación. Una aproximación didáctica a la determinación de modelos mentales en la formación de profesores en química}

Rafael Yecid Amador Rodríguez

\section{Descripción}

En este trabajo de investigación se propone una estrategia didáctica innovativa para determinar modelos mentales explicativos sobre el concepto de combustión.

\section{Resumen}

Se presentan los resultados de investigación que se obtuvieron a partir del diseño de una estrategia didáctica, encaminada a modificar los modelos mentales explicativos de los estudiantes, con miras a lograr un acercamiento al discurso de la comunidad científica, en particular al concepto de combustión desde las propuestas de Stahl y Lavoisier, estrategia que cifra su importancia en las relaciones entre la didáctica de la química, la formación inicial de profesores de química y la historia de la química.

\section{Metodología}

La metodología se derivó del marco conceptual, en el que se identificó aquel conocimiento que ha construido cada estudiante acerca del concepto de combustión. Para ello se utilizaron las pruebas tipo Likert, de composición, y los conversatorios. Planteado el marco conceptual y metodológico, se explicita a continuación la estrategia didáctica con 
que se realizó la investigación. Aplicación de las pruebas tipo Likert. Lectura de los documentos que presentan menos deformación conceptual de la propuesta de Georg Ernst Stahl para el concepto de combustión por parte de los profesores en formación inicial, en cuanto al modelo del flogisto. Elaboración de composiciones a cargo de los estudiantes (trabajo individual). Discusión en el colectivo aula de la propuesta de Georg Ernst Stahl. Elaboración de composiciones por parte de los estudiantes (trabajo grupal).

Lectura de los documentos que presentan menos deformación conceptual de la propuesta de Antoine Laurent Lavoisier. Elaboración de composiciones por parte de los estudiantes (trabajo individual). Discusión con el colectivo aula en torno a la propuesta de Antoine Laurent Lavoisier. Elaboración de composiciones por parte de los estudiantes, trabajo grupal.

\section{Conclusiones}

La investigación que se hizo en el espacio académico de teorías químicas I permitió formular una estrategia didáctica no convencional, esto es, se diseñó la estrategia desde los presupuestos constructivistas, que parten del convencimiento de que la reconstrucción y la construcción del conocimiento son un proceso colectivo y no individual. En la metodología de enseñanza la historia de la química, específicamente del concepto de combustión, cumplió un papel importante, ya que los profesores en formación se remitieron a la historia para hacer de sus composiciones. Se afirma que dicha estrategia fue exitosa, ya que se evidenciaron cambios en los modelos mentales explicativos.

Ha de aclararse que teorías químicas I es un espacio académico prescrito en el programa de licenciatura en química, en el que el conocimiento químico se hace objeto de trabajo y la epistemología y la historia no constituyen los intereses primordiales del mismo. Vistos los análisis de resultados de entrada y salida de las Pruebas tipo Likert, podríamos afirmar que hubo cambios -aunque no significativos- en las concepciones epistemológicas y didácticas. Se parte del convencimiento de que transformar las concepciones epistemológicas de estos futuros profesores en formación no es un proceso inmediato, ya que demanda tiempo y esfuerzo tanto de los profesores en formación como de los profesores en ejercicio. Vale anotar que pese a que el trabajo de investigación se hizo sólo durante cuatro semanas, se aspira a que posteriores trabajos continúen en la misma dirección, por sus resultados, con el propósito de que se consolide un proceso investigativo que permita una mayor interacción en el tiempo con los estudiantes y así evidenciar tanto las bondades como las falencias de la propuesta.

Respecto de lo didáctico, cabe destacar un cambio conceptual y metodológico de los profesores en formación inicial en química, en cuanto abandonan la concepción de que la didáctica es la parte instrumental de la pedagogía; esta afirmación surge de los resultados obtenidos a partir de la prueba tipo Likert. Se encuentra que los profesores en formación inicial en química le atribuyen importancia a la historia de las 
ciencias en la enseñanza de las mismas, lo que se reflejó evidentemente en las composiciones. El involucrar la historia de la química -en especial la de la construcción del concepto de combustión- en las composiciones elaboradas por los futuros profesores de química les permitió conocer los problemas y las dificultades que enfrentaron quienes elaboraron el concepto de combustión y, por ende, acercarse a lo que la comunidad ha propuesto al respecto.

Realizado el trabajo didáctico, se asevera que los modelos mentales explicativos de los profesores en formación inicial en química se van modificando y acercando a los consensos de la comunidad científica. Por tal motivo, se afirma que los modelos mentales explicativos que construyen los futuros profesores en formación no son estáticos, lo que permite concluir que el proceso de aprendizaje, a más de dinámico, no es lineal ni acumulativo. En lo que respecta a las composiciones, vale la pena señalar que se evidencia una reconstrucción y construcción de los modelos mentales explicativos y que dichos modelos, en gran parte están soportados desde los presupuestos del constructivismo. $\boldsymbol{\Delta}$

\title{
Estrategia didáctica para la enseñanza-aprendizaje por investigación del enlace químico a partir de la elaboración de modelos moleculares por computador
}

\author{
Álvaro Pío Rojas Duarte
}

\section{Descripción}

La propuesta de investigación se aplicó a una población de 32 estudiantes de décimo grado del IED Miguel Antonio Caro, localizado en la localidad décima (Engativá) de Bogotá. El trabajo consistió en la implementación de una guía de actividades, concebida desde el modelo de enseñanza y aprendizaje por investigación, complementada con entorno virtual (software) diseñado para el tema y articulado con las actividades para lograr que los estudiantes construyan conocimiento en todo lo concerniente al enlace químico y sus implicaciones en la estructura molecular de algunas sustancias.

\section{Resumen}

El proyecto surgió teniendo en cuenta los siguientes referentes: las disertaciones realizadas en el Programa de Maestría en Docencia de la Química, las reflexiones hechas en torno a las implicaciones educativas de las nuevas tecnologías de la información y la comunicación en los procesos de enseñanza y aprendizaje de la química, y los principios epistemológicos, psicológicos y metodológicos del modelo constructivista de enseñanzaaprendizaje por investigación. Se describen la evolución conceptual, así como el cambio metodológico y actitudinal, los cuales se basan en la experimentación de la guía de actividades. Por otra parte, 
se muestran el diseño y la aplicación de una estrategia didáctica que favoreció el aprendizaje significativo del concepto enlace químico y sus implicaciones en la estructura molecular de algunas sustancias, aplicando un modelo de intervención en el aula de enseñanza de las ciencias por investigación.

\section{Metodología}

Con base en lo anterior se desarrolló el presente trabajo, que tuvo como finalidad la enseñanza del concepto enlace químico a partir de la implementación de una guía de actividades apoyada por computador, fundamentada en el modelo de enseñanza y aprendizaje por investigación. Dicho programa de actividades se elaboró en función de la revisión bibliográfica efectuada sobre el tema. Se consideró de vital importancia someter a prueba esta propuesta, que gira en torno a un concepto estructurante vital para la comprensión de la disciplina.

Se planteó como parte de la estrategia el tratamiento de diversas situaciones, que tratan de familiarizar a los estudiantes con la metodología científica, lo que permitió superar el reduccionismo de los modelos de cambio conceptual que se centran exclusivamente en los contenidos conceptuales. Por ello, en la estrategia se enfatiza en que el aprendizaje requiere, de acuerdo con el modelo constructivista, la actividad autoconsciente por parte del sujeto como condición para su evolución intelectual, dado que quien aprende interpreta la realidad proyectando sobre ella los significados que va construyendo. Se presentaron problemas interesantes y pertinentes para los estudiantes. Así, la construcción de conocimientos no se planteó como un cuestionamiento de las ideas de los estudiantes, sino como el resultado de las investigaciones realizadas por ellos para resolver problemas. (Gil et al., 1991).En este proceso surgieron criterios y razones no arbitrarias para decidir si las ideas aportadas se aceptaban o no como un avance. De esta manera, una enseñanza por investigación que involucra la metodología científica pudo integrar el desarrollo de contenidos específicos con elementos metodológicos, de manera que se avanzó en la construcción de conceptos científicos.

Los contenidos específicos fueron los siguientes: 1) Estructura de la materia, por la gran dificultad que tienen los estudiantes para explicar la naturaleza de las sustancias y algunos de sus cambios observables (Stavy, 1988; Bar, 1989). 2) El enlace químico, considerado por muchos investigadores un concepto crucial dentro de la química (Benfey, 1965; Allinger et al., 1979; Paoloni, 1979; Langmuir, 1921, recogido en Jensen, 1984; Solbes y Vilches, 1991; Pauling, 1992, entre otros). 3) Niveles de representación mental. La propuesta de Johnstone $(1982,1991)$ para la comprensión de las ciencias naturales en general y de la química en particular, niveles macroscópico, submicroscópico y simbólico, este último enfocado a la geometría y a la polaridad molecular de algunas sustancias. Teniendo en cuenta que estos conceptos, como tantos otros en química, requieren un buen nivel de abstracción ligado a la generación de modelos, se consideró la incorporación de elementos multimediales; es el caso del software elaborado con programas de diseño gráfico, visualizadores 
moleculares y videos, para posibilitar desde lo tridimensional el aprendizaje de dichos conceptos, haciendo uso de modelos analógicos y representacionales, de tal manera que el estudiante pudo resolver diferentes situaciones manejando simultáneamente los tres niveles representacionales propuestos por Johnstone para explicar un fenómeno químico.

La población de la investigación -de tipo cualitativa experimental- estuvo constituida por un grupo experimental. Para verificar el alcance de la propuesta se aplicaron instrumentos pretest y postest, al igual que la guía de actividades, distribuidos en tres fases. La primera constaba de tres etapas: 1) Diseño y validación de los instrumentos que se van a utilizar para la contrastación de las hipótesis. 2) Diseño, ejecución y evaluación de las guías de actividades sobre el tema del enlace. 3) Diseño del software, que contribuye al mejoramiento del aprendizaje de la química. La segunda fase se llamaba Aplicación y experimentación, y la tercera fase se denominaba Presentación y análisis de resultados, donde se categorizaron las preguntas del pretest y postest en los temas: conceptos básicos, tipos de enlace y teorías de enlace y geometría molecular.

El diagnóstico se hizo mediante la aplicación del pretest, el cual permitió establecer las ideas previas de los estudiantes y el nivel de conocimientos de las temáticas que había que trabajar; con base en esta información se pudieron verificar los prerrequisitos y se realizaron las actividades de nivelación pertinentes; la etapa de implementación de la guía de actividades, organizada en secuencias de iniciación, de desarrollo y finalización, creó las condiciones para la construcción del conocimiento a partir de la solución de situaciones presentadas, complementadas con el apoyo del software. Por último, se efectuó validación, donde se incluyó el postest, que permitió determinar el alcance de la propuesta con respecto a los objetivos establecidos.

\section{Conclusiones}

La guía de actividades titulada "Enlace químico y estructura de la materia”, basada en el modelo de enseñanza-aprendizaje por investigación que involucra la incorporación de NTIC en la enseñanza de la química, es una poderosa estrategia didáctica que favorece el desarrollo de las estructuras conceptuales, metodológicas, estructurales y axiológicas de los estudiantes, enriqueciendo su formación personal, su nivel de comunicación, expresión, dinamismo, compañerismo, responsabilidad, lo que se traduce en un cambio de actitud mental que además de posibilitarle el aprendizaje de conocimientos, reconoce la importancia de la asignatura en su entorno natural inmediato.

Encontrar nuevas estrategias que el profesorado pueda adecuar a sus prácticas y que favorezcan los aprendizajes del alumnado.

Las simulaciones virtuales permiten un mejor aprendizaje, sobre todo en aquellos aspectos donde la experimentación real no es posible. Hacer más atractivo el estudio de la química, aumentando así la motivación del alumnado.

De acuerdo con los resultados obtenidos en el pretest y el postest, se pudo determinar que se presentó un 
incremento porcentual en las categorías establecidas para el estudio y análisis de los resultados: mayores porcentajes de respuestas correctas en los estudiantes en el postest. A pesar de lo abstracto y denso del tema de geometría molecular para los estudiantes de secundaria, los resultados del pretest ubicaron a 22 estudiantes en la categoría Medio (69\%), cuatro en la categoría Alto (13\%), seis estudiantes en categoría Bajo (19\%); los resultados de geometría molecular en el postest ubican a 17 estudiantes en categoría Alto (53\%), 14 estudiantes en categoría Medio (44\%) y un estudiante en categoría Bajo (1\%). Es una de las razones por las cuales se puede concluir que un programa de actividades, complementado con apoyos informáticos, es una excelente estrategia para la enseñanza del enlace químico, que requiere algunos ajustes para mejorar el nivel de aprendizaje y llevarlo a una categoría Alta.

Con la implementación de esta estrategia se confirma la importancia del modelo de enseñanza y aprendizaje por investigación, las guías de actividades con situaciones planteadas y apoyo virtual como complemento, estrategia válida para la enseñanza de la química en estudiantes de educación media.

De igual manera se pudo establecer que, como lo afirma Fortman (1992), los modelos moleculares como modelos analógicos son necesarios en química porque la enseñanza de esta ciencia conlleva un gran componente de razonamiento abstracto y estos modelos pueden ayudar al aprendizaje no sólo desde un punto de vista cognitivo sino porque también tienen un efecto de motivación (Thiele y Treagust, 1994).

Por último, hay que tener en cuenta que, de acuerdo con Lawson et al. (1993) y Gabel (1998), para que se favorezca el cambio conceptual se requiere que los estudiantes tengan ciertas destrezas hipotético-deductivas, las que se pueden verificar a partir de la solución de situaciones problemáticas, es decir, de su capacidad para emitir hipótesis y consecuencias derivadas. $\boldsymbol{\Delta}$

\section{Maestría en Tecnologías de la Información Aplicadas a la Educación}

Universidad Pedagógica Nacional, Departamento de Tecnología

Atención y sensibilidad rítmica en niños con limitación auditiva

Adriana Leiva Bustos

\section{Descripción}

Con esta investigación se busca definir modelos que incorporen el uso de las TIC y satisfagan las necesidades educativas de las personas con limitación auditiva, en lo pertinente a la formación rítmica y al desarrollo de habilidades menta- les que ésta genera, centrándose en la atención, en tanto que gran parte de la historia de la educación de los sordos se ha soportado en el modelo clínico y la visión del sordo como persona deficiente. De igual manera, en las instituciones 
educativas cuya visión se centra en la atención de este tipo de población, se desarrollan métodos encaminados a la rehabilitación auditiva y a la enseñanza de la lengua castellana. Pero un aspecto aún más preocupante es que en las propuestas educativas para sordos se obvia a esta comunidad, por lo cual se parte de estrategias pensadas y hechas para oyentes. Su objetivo general pretende identificar las diferencias existentes en los niveles de atención y en el desarrollo de la sensibilidad rítmica entre un grupo de niños con limitación auditiva, entrenado por un ambiente computacional que incorpora el Lenguaje Visual (LV) por medio del Lenguaje de la Imagen Animada (LIA), y otro con las mismas características, entrenado por un ambiente que incorpora el Lenguaje Icónico (LI) a través de la Lengua de Señas (LS). En el diseño de la propuesta incorpora la unidad didáctica para guiar la metodología de la formación rítmica en los dos ambientes computacionales desarrollados.

\section{Contenidos}

El estudio se empieza a desarrollar a partir de la descripción del problema que aborda su caracterización, antecedentes, justificación, objetivos y pregunta de investigación, para pasar al segundo capítulo, en el que se fundamentan teóricamente los elementos conceptuales tratados. En el tercer capítulo se conceptualiza el dominio: el ritmo, la formación de la sensibilidad rítmica y el ritmo en el mundo. Posteriormente, en el cuarto capítulo, se define el modelo de la investigación; en el quinto se determina el diseño metodológico, donde se precisan el diseño de la investigación, las técnicas e instrumentos de medición aplicados, las hipótesis y la especificación de variables, la validación del ambiente computacional y la descripción del mismo. Finalmente, en el sexto capítulo, se realiza el análisis e interpretación de datos a nivel cuantitativo y, como resultado de este estudio, se elabora un modelo pedagógico para la enseñanza rítmica de limitados auditivos.

\section{Conclusiones}

El estudio del análisis cuantitativo permitió evidenciar que el ambiente computacional que incorpora el Lenguaje Icónico por medio de la Lengua de Señas genera mayores niveles de atención y de sensibilidad rítmica, con respecto al ambiente computacional que incorpora el Lenguaje Visual, por medio de la imagen animada. Así mismo, se determinó que sí existen diferencias significativas. El ambiente computacional que incorpora el Lenguaje Icónico, genera un mayor nivel de atención y de formación de la sensibilidad rítmica. Por otra parte, al hacer referencia al grado de relación existente entre las dos variables objeto de experimentación, no se encuentra que a un mayor nivel de atención corresponde un mayor desarrollo de la sensibilidad rítmica, lo cual indica que entre las variables atención y sensibilidad rítmica no se halla base de correlación. Aunque se concluye que el ambiente computacional que incorpora el Lenguaje Icónico es el que genera en forma significativa un mayor nivel de atención y de formación de la sensibilidad rítmica, así mismo se encuentra que el grado de relación existente entre las dos variables es débil, por lo que la formación de la sensibilidad rítmica no depende del nivel de atención. $\boldsymbol{\Delta}$ 


\section{Aprendizaje en la solución de problemas sobre rotación espacial entre grupos de estudiantes que interactúan con un sistema hipertexto y un tutor inteligente}

Héctor Mauricio Díaz Restrepo

\section{Descripción}

En la presente investigación se analiza la incidencia que el tratamiento de dos ambientes informáticos de aprendizaje, Hipertexto y Tutor Inteligente, puede ejercer en el proceso de aprendizaje en la solución de problemas de rotación espacial de formas bidimensionales. La muestra del estudio estuvo conformada por 54 estudiantes del Colegio Nacional Superior de la ciudad de Cartago, divididos en dos grupos experimentales. Se plantea la hipótesis de que el grupo de estudiantes que se entrene con el sistema Tutor Inteligente obtendrá un desempeño mejor en la solución de problemas de rotación espacial, medido en términos de eficiencia y eficacia, que los estudiantes que se entrenen con el sistema Hipertexto. El análisis de los resultados de las pruebas estadísticas confirma la hipótesis experimental.

\section{Contenido}

En el primer capítulo se explica el planteamiento del problema de investigación, basado previamente en una justificación que da origen al mismo, al tiempo que se describen los objetivos que se persiguen en el estudio.

En el segundo capítulo se formulan los antecedentes o estado del arte que soportan el estudio y avalan el problema de investigación. En este apartado se pretende dar a conocer las investigaciones que se han realizado previamente en el campo computacional sobre pruebas de rotación espacial, y la incidencia que estos estudios han tenido sobre el desempeño de solución de problemas en términos de eficiencia o eficacia. En el tercer capítulo se explica el dominio de conocimiento sobre el cual se desarrolló esta investigación. La rotación mental, la habilidad espacial, la representación del espacio, los tutores inteligentes y los hipertextos son los temas más relevantes. Allí se encuentran implícitos los modelos de aprendizaje de los sistemas hipertextos y los tutores inteligentes, sustentados en algunos mapas conceptuales que explicitan la adquisición del conocimiento.

El cuarto capítulo se centra en el diseño experimental y la metodología usada en la investigación. Se determinan las hipótesis y variables que intervienen en la investigación. Se define la población de trabajo y se explica detalladamente el proceso de conformación de los grupos experimentales. El capítulo quinto está dedicado a describir cada uno de los dos ambientes de aprendizaje computarizados que se emplearon en la etapa de entrenamiento de los dos grupos, el Hipertexto y el Tutor Inteligente. Se detallan la forma como se debe interactuar con los programas y el funcionamiento de los módulos de entrenamiento. Igualmente, se detalla el software usado para la etapa de eva- 
luación, el cual es el mismo para ambos grupos experimentales. En el capítulo sexto se detallan los resultados de las pruebas estadísticas efectuadas durante el estudio, con el fin de confrontar la hipótesis experimental.

En el capítulo séptimo se analizan e interpretan los resultados del capítulo anterior. El principal hallazgo encontrado apunta a validar la hipótesis experimental, confirmando que en la solución de problemas de rotación espacial los estudiantes entrenados con el sistema Tutor Inteligente se desempeñan mucho mejor que aquellos que se entrenaron con el sistema Hipertexto. Los resultados también apoyan las teorías de algunos investigadores que se tratan en los antecedentes.

El capítulo octavo está dedicado a explicar las conclusiones más importantes halladas durante el estudio, basadas en la interpretación y análisis de los resultados y soportadas en los antecedentes y marco teórico de la investigación. Se hace además un aporte pedagógico y se deja al lector la inquietud de poder profundizar y realizar otro tipo de investigaciones en el campo de la rotación espacial, usando otros ambientes de aprendizaje computarizados. Al final, se encuentra la bibliografía que soporta el presente estudio.

\section{Metodología}

El tratamiento que permite trabajar mejor la pregunta de investigación y el desarrollo de los objetivos es el experimental. Se trata de un diseño experimental con grupos aleatorizados y sólo posprueba. Para validar la hipótesis se realizan pruebas $\mathrm{T}$ Student entre dos grupos experimentales, expuesto cada uno a un ambiente computarizado diferente, un sistema Hipertexto y un Tutor Inteligente, con el objetivo de prepararlos para una prueba de rotación espacial. Se tiene una variable independiente: Sistema Informático de Aprendizaje, representada en dos niveles: Tutor Inteligente e Hipertexto. La variable dependiente es el Aprendizaje, medida en términos de eficiencia y eficacia.

\section{Conclusiones}

Entre las principales conclusiones se encuentran las siguientes.

La interpretación y el análisis de resultados permiten aceptar la hipótesis experimental, es decir, se afirma que existen diferencias significativas entre los puntajes mostrados tanto en eficiencia como en eficacia, debido a la manipulación de la variable independiente. Las diferencias encontradas en los resultados de las pruebas en términos de eficacia apoyan las teorías de Dixon (1997), que sustenta que ambientes de aprendizaje como los tutores inteligentes que utilizan instrucciones dinámicas y módulos de procesamiento de información basados en técnicas de la imagen animada, mejoran en los estudiantes las estructuras cognitivas sobre pensamiento geométrico espacial.

Del análisis de los resultados se pudo deducir que en general los estudiantes fueron más eficaces (acertados) en la solución de problemas sobre rotación espacial cuando el patrón de rotación de los estímulos se encontraba en el sentido derecho, que cuando está en sentido izquierdo. El tipo de estímulo de matriz (nivel 1 o nivel 2) utilizado durante las pruebas no influyó sobre el rendimiento en la solución de los problemas, tanto en 
eficiencia como en eficacia para ambos grupos del experimento. De esta manera, los resultados son acordes con los hallados por Cohen y Kubovy (1993), donde el grado de complejidad expuesto en los tipos de matrices en las pruebas no incidió significativamente en los valores dados por el tiempo de reacción. $\boldsymbol{\Delta}$

\section{Reportes automatizados y verbales: incidencia en el aprendizaje de la solución de problemas}

Nilson G. Valencia Vallejo

\section{Descripción}

Como propósito general, se busca analizar la incidencia que tienen los reportes automatizados y verbales sobre el aprendizaje, medido en términos del número de eventos realizados y el tiempo empleado en la solución de problemas de palancas de primer género.

\section{Contenidos}

En el primer capítulo se plantean los parámetros generales que orientan el desarrollo de la investigación, así como las investigaciones que dan origen a este trabajo. En el segundo capítulo se presentan los referentes teóricos asumidos en el estudio, entre estos Micromundos computacionales y la construcción de conocimiento, Estrategias cognoscitivas, Resolución de problemas: fundamentos cognitivistas, Feedback y La autorregulación en los procesos de solución de problemas. En el capítulo tercero se habla sobre el dominio de conocimiento, referido específicamente a la temática tratada en el ambiente de aprendizaje computacional. En el capítulo cuarto se muestra el modelo elaborado para la investigación. En el capítulo cinco se presenta el marco metodológico abordado para dar validez a las hipótesis planteadas. En el capítulo seis se sustentan los resultados obtenidos a partir del análisis de varianza de medidas repetidas y los análisis cualitativos elaborados para complementar los resultados estadísticos. También se explica la visión general de los resultados encontrados con respecto a las variables planteadas. Finalmente, se presentan las conclusiones, resaltando el aporte teórico, la relación pedagógica y las proyecciones de este estudio.

\section{Conclusiones}

En general, las pruebas estadísticas realizadas arrojaron muy pocas diferencias significativas entre ambos tipos de retroalimentación. Si bien esto no implica una confirmación de que en los procesos cognitivos operen con eficacia y eficiencia similar, estos resultados no obligan a descartar tal semejanza. Es posible que dicha heterogeneidad del comportamiento de los sujetos en las tres muestras -observable en los valores altos de las varianzas- haya contribuido a restar significación a ciertas tendencias divergentes, de todas maneras constatadas. 
Algunas de las pruebas, sin embargo, ofrecen respuestas interesantes a las subpreguntas en que se particularizó la cuestión general. Por ejemplo:

Estadísticamente no se observan diferencias significativas frente al hecho de usar un tipo de intervención particular (feedback adaptativo) sobre el número de eventos empleado y el tiempo utilizado en la solución de problemas. Pero se observa un avance progresivo paralelo en los tres grupos frente a estos hechos, sobre todo en el grupo que incorpora simuladores de procesos. Situación que convalida las conclusiones de trabajo de Maldonado et al. (2001), quienes afirman que en la medida en que los sujetos solucionan problemas, consolidan o desarrollan estrategias más fuertes, que conducen a mejores resultados en términos de eficiencia y eficacia.

El grupo A (intervención automatizada) evidencia un impacto más fuerte sobre la disminución del número de eventos realizados en la solución de problemas, que el grupo B (intervención auditiva) y el grupo C (sin intervención). Lo anterior permite revalidar las afirmaciones hechas por Maldonado et al. (2001), quienes plantean que existe un impacto fuerte en el uso de simuladores de procesos sobre la eficacia. El hecho de ver reflejado su propio comportamiento en un dispositivo objetiva los propios procesos de aprendizaje y motiva al estudiante a construir conocimiento sobre su propio conocimiento, es decir, a desarrollar metacognición.

En este mismo orden de ideas, el grupo que incorpora el uso de simulaciones en sus procesos, al mostrar una disminución más fuerte en el número de eventos y el tiempo empleado, per- mitiría relacionar ambos hechos con la pauta de un mayor control de su proceso de aprendizaje. Esta afirmación se sustenta también en los estudios realizados por Oxford y Crookall (1988), quienes afirman que las simulaciones fomentan el desarrollo de estrategias de aprendizaje, e incluyen estrategias organizacionales, estrategias afectivas estrategias de memoria y otras. Este aspecto se evidencia en lo manifestado por el grupo A en lo que concierne a su mayor nivel.

En el grupo que interactúa con la audición de sus reportes (grupo B) se evidencia un efecto importante en relación con su comportamiento en términos de las metas planteadas (gráfica 6.5). Esto refleja un efecto marcado positivamente en la autorregulación (Akhras \& Self, 1997) de sus procesos, en comparación con los otros dos grupos. Aunque este hecho no permitió relacionar una mejoria sobre el número de eventos realizados y el tiempo empleado, atribuida posiblemente a la falta de interacción con el modelo de aprendizaje planteado, cabe proponer una hipótesis complementaria: la audición de los reportes verbales propios en la solución de problemas de palancas de primer género genera un efecto positivo sobre la planeación, monitoreo y evaluación de procesos propios: autorregulación (Bandura, 1991). Vale la pena mencionar que este hecho se evidenció en el análisis cualitativo, los sujetos que escuchan sus reportes expresan control en sus acciones y se acercan con mayor éxito al modelo gráfico-matemático de solución de los problemas planteados, lo que incide en la utilización de mayor tiempo por los procesos de control realizados sobre 
sus acciones. Éstos recurren en menor medida a movimientos aleatorios.

El hecho de que no se refleje incidencia en la variable grupo, en el análisis de medidas repetidas, permite afirmar que la intervención del feedback adaptativo, aplicado a los grupos experimentales al finalizar los procesos de solución de problemas, no presentó un contenido óptimo en cuanto a la demanda cognitiva de los problemas planteados, el desarrollo de habilidades cognitivas, el conocimiento previo y la motivación del alumno. Lo anterior se ve reflejado en la diversidad de los resultados tanto entre grupos (varianza sistemática) como dentro de éstos (varianza de error). Esta afirmación se sustenta a partir de Smith (1988), quien concibe el diseño de la instrucción como un acto de balance entre proporcionar a los estudiantes un soporte suficiente como para que puedan aprender y preservar en su tarea, y el límite a tal soporte, de modo que ellos puedan acceder a la profundidad requerida en el procesamiento de dicho aprendizaje.

Finalmente, con base en los planteamientos de Ainsworth (1999), cabe pensar que la suma de las representaciones múltiples (gráficas y tablas) incorporadas en el diseño del ambiente (interfaz), así como sus conexiones, no beneficiaron con sus propiedades a los estudiantes en virtud de sus múltiples ventajas en el aprendizaje, por cuanto éstos tenían que traducir estas relaciones, hecho que implicó un mayor esfuerzo cognitivo. A pesar de que dichas relaciones se daban automáticamente por la conexión dinámica del ambiente, éstas debieron ser más explícitas, tal vez a través de señales visuales o sonoras, como resaltando sus componentes principales. Esta evidencia se produce más por el proceso de observación del investigador y el análisis cualitativo realizado que por los resultados estadísticos, que de alguna manera se vieron reflejados en éstos. $\Delta$ 